\title{
Minimally Invasive Mediastinal Parathyroidectomy: An Effective and Safe Technique in Patients with Ectopic Primary Hyperparathyroidism
}

\author{
Iskander Al-Githmi \\ Department of Surgery, Division of Cardiothoracic Surgery, King Abdulaziz University Hospital, Jeddah, KSA \\ Email: algithmi@hotmail.com
}

How to cite this paper: Al-Githmi, I. (2017) Minimally Invasive Mediastinal Parathyroidectomy: An Effective and Safe Technique in Patients with Ectopic Primary Hyperparathyroidism. Open Journal of Thoracic Surgery, 7, 70-76.

https://doi.org/10.4236/ojts.2017.74010

Received: December 4, 2017

Accepted: December 25, 2017

Published: December 28, 2017

Copyright $\odot 2017$ by author and Scientific Research Publishing Inc. This work is licensed under the Creative Commons Attribution International License (CC BY 4.0).

http://creativecommons.org/licenses/by/4.0/ (c) (i) Open Access

\begin{abstract}
Introduction: Primary hyperparathyroidism is a disease commonly caused by a single parathyroid adenoma in $80 \%-85 \%$ of cases, and is less frequently caused by parathyroid hyperplasia. In approximately $2 \%$ of cases, the ectopic parathyroid adenoma is located within the mediastinum. Safely targeted parathyroidectomy is the gold standard procedure for surgical management of primary hyperparathyroidism. We reviewed our surgical experience using video-assisted thoracic surgery and a robotic technique for surgical resection of mediastinal parathyroid adenoma, caused by primary hyperparathyroidism. Materials and Methods: From July 2010 to September 2016, six consecutive patients with symptomatic primary hyperparathyroidism were evaluated with neck ultrasound computed tomography (CT), technetium-99 sestamibi scintigraphy, and selective venous sampling with a measurement of parathyroid hormone levels. Four patients underwent video-assisted thoracic parathyroidectomy and two patients had robotic-assisted parathyroidectomy. Results: In six patients, there were four women and two men with a mean age of 47.5 years (ranging from 31 - 60 years). The mediastinal parathyroid adenomas were successfully localized by preoperative imaging studies in five patients. Only in one patient, we were unable to localize the parathyroid adenoma. All parathyroid adenomas were successfully resected without any complications. Conclusions: We found that minimally invasive mediastinal parathyroidectomy is promising, safe, and effective in a selected group of patients, as well as providing superior cosmetic results and a short hospital stay.
\end{abstract}

\section{Keywords}

Primary, Hyperparathyroidism, Parathyroid Adenoma, Minimally Invasive, 
Parathyroidectomy, Mediastinum

\section{Introduction}

Primary hyperparathyroidism is an endocrine disease characterized by excess production of parathyroid hormone, resulting in hypercalcemia. It is caused by a single parathyroid adenoma in $85 \%$ of cases. Typically, the culprit adenoma is located in the cervical region; however, $15 \%-20 \%$ of parathyroid adenomas are ectopically located, with only $1 \%-2 \%$ located in the anterior mediastinum. Other locations include the aortopulmonary window, the para- or retroesophageal position, and that along the carotid sheath. However, minimally invasive mediastinal parathyroidectomy, such as video-assisted thoracic surgery and robotic-assisted surgical techniques, is gaining popularity because of a number of advantages over sternotomy and thoracotomies: these also include superior cosmetic results, less postoperative pain, shorter hospital stay, and rapid recovery compared to open conventional surgical approaches. The aim of this study is to review our experience of minimally invasive mediastinal parathyroid adenoma, which was successfully resected utilizing robotic and video-assisted thoracic surgery.

\section{Materials and Methods}

Subjects included six patients with primary hyperparathyroidism. 2 of whom underwent robotic parathyroidectomy and the remaining 4 underwent video-assisted thoracoscopic parathyroidectomy from July 2010 to September 2016. Prior neck exploration was performed in one patient.

\subsection{Inclusion Criteria}

Six patients admitted with primary hyperparathyroidism were included in the study. There were four women and two men, with a mean age of 47.5 years (range, 31 - 60 years).

\subsection{Preoperative Localization}

All patients received cervical ultrasounds, double-phase technetium-99m sestamibi (Tc-99m-sestamibi) scintigraphy along with Single Proton Emission Computerized Tomography (SPECT) [Figure 1], and enhanced-neck and chest CT [Figure 2]. Selective venous sampling for parathyroid hormone measurement was performed in one patient when preoperative parathyroid adenoma localization failed.

\subsection{Surgical Management}

Four patients received video-assisted thoracic surgery and two patients underwent robotic-assisted surgery, which were performed under general anesthesia 


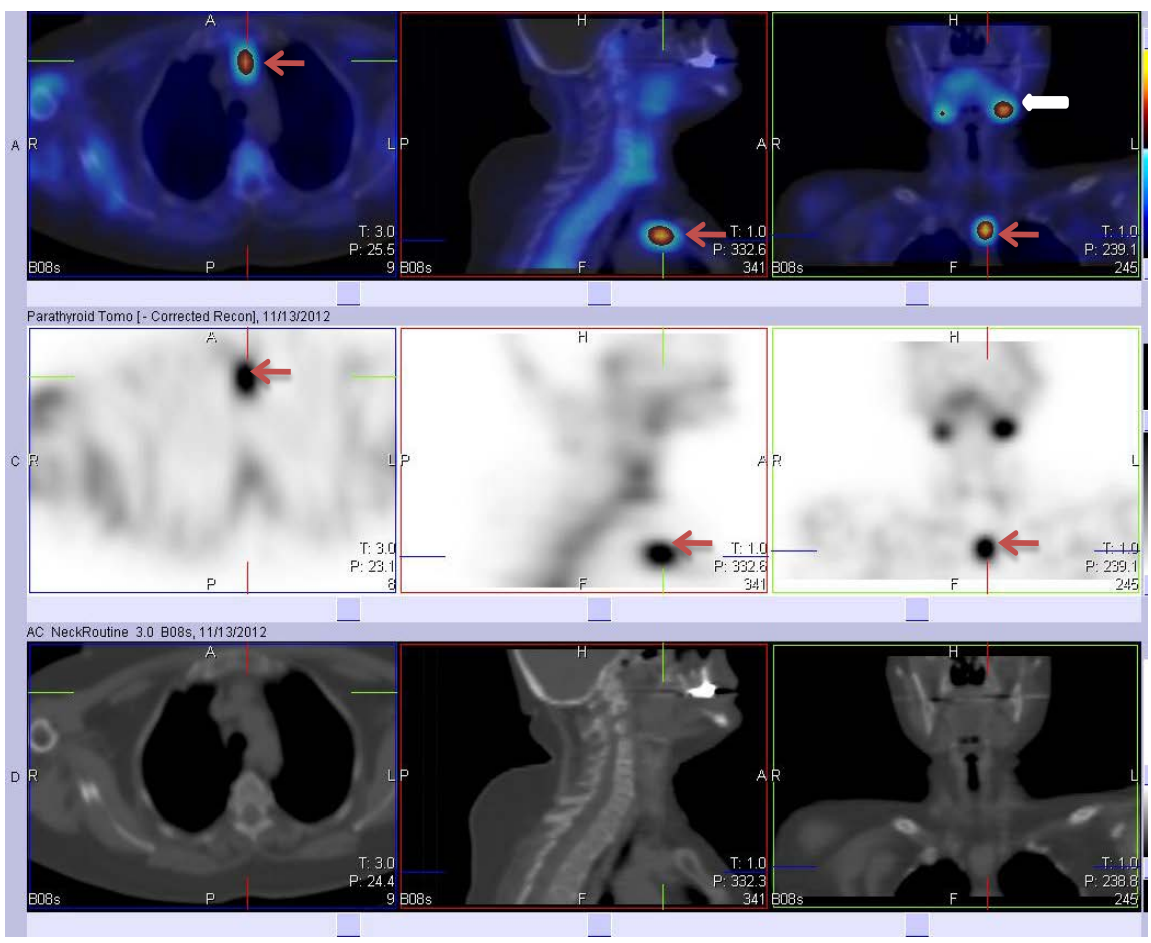

Figure 1. Single-photon emission computed tomography/computed tomography scan with technetium $99 \mathrm{~m}$ sestamibi shows increased uptake at the parathyroid adenoma (red arrows) and left submandibular salivary gland (white arrow).
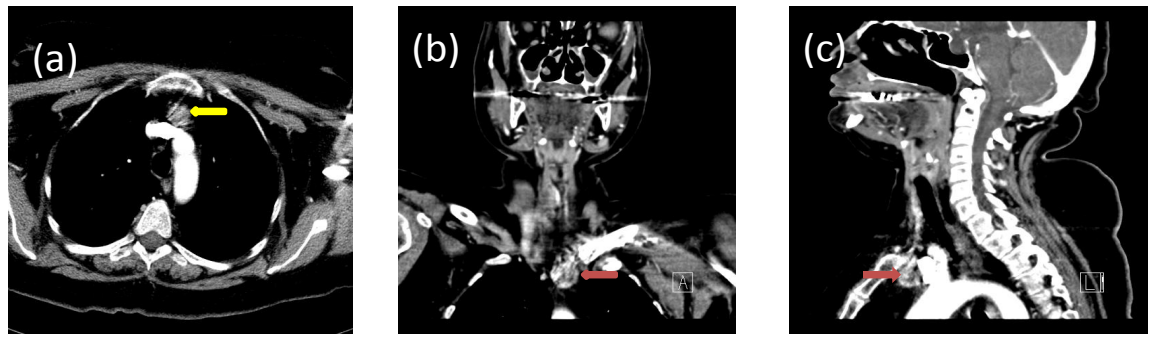

Figure 2. Computed tomography scan: (a) Axial image shows ectopic parathyroid adenoma (yellow arrow) above the left brachiocephalic vein. (b), (c) Coronal and sagittal images show the ectopic parathyroid adenoma (red arrows).

with double lumen endotracheal intubation. The patient was placed in a supine position with the arm positioned as far back as possible to gain enough space. Three skin incisions were made along the sub-mammary fold; the camera port (30-degree angled lens) was introduced through a fifth intercostal space along the anterior axillary line. The right and left arms ports were introduced through a fifth intercostal space, midclavicular, and then through a third intercostal space, mid-axillary line. The point of reference to the anterior mediastinum was the phrenic nerve. The left arm port used an Endo-Wrist (Sunnyvale, CA, USA) precise grasper; the right arm port used an Endo-Wrist harmonic shear dissector. Dissection started and extended superiorly along the phrenic nerve to the jugular fold and continued inferiorly along the retrosternal area. The dissection con- 
tinued to where the parathyroid adenoma was identified. The adenoma gland and mediastinal thymic tissue were removed en bloc, placed in an endo-bag, retrieved through a trocar incision, and sent for frozen section. Patient blood samples were collected by the anesthesiologist 10 minutes after removal of the suspected abnormal gland and sent for a parathyroid hormone assay. We saw a drop in parathyroid hormone level of more than $50 \%$, compared to the highest preoperative parathyroid hormone occurring during surgery, which suggested that all hyperfunctioning glandular tissue was removed.

\subsection{Patient Follow-Up}

All six patients were followed-up in our outpatient clinic for 12 months. At each visit, PTH assays and serum calcium levels were assessed.

\section{Results}

Mediastinal parathyroid adenomas were identified by preoperative localization scanning methods in five patients. A selective venous sampling was used to identify hyper-functioning glandular tissue in one patient who had negative preoperative scanning techniques. During the course of the pre-surgical procedure in regards to parathyroid hormone measurement, all patients' hormonal levels were significantly elevated, with mean level of $88.9 \mathrm{pg} / \mathrm{ml}$. The clinical data and patient characteristics are summarized in Table 1 . The mean operative time was 149 minutes, while the average hospital stay was 3.8 days. There were no operative or postoperative complications. Ectopic mediastinal parathyroid

Table 1. Patient characteristics.

\begin{tabular}{cc}
\hline Patient characteristics & Number $=6$ \\
\hline Age, mean (years) & 47.5 \\
Gender, no. (\%) & $2(34)$ \\
Male & $4(66)$ \\
Female & \\
Preoperative biochemical level & 88.9 (range, $16.3-19.4)$ \\
PTH (pg/ml) & 2.72 (range, $1.7-3.5)$ \\
Calcium (mmol/ml) & \\
Preoperative localization & 5 \\
Positive & 1 \\
Negative & 1 \\
Prior neck exploration & \\
Surgical approach & \\
VATS & 2 \\
Robotic & \\
\hline
\end{tabular}

PTH = parathyroid hormone; VATS = video-assisted thoracic surgery. 
Table 2. Postoperative results.

\begin{tabular}{cc}
\hline Patient characteristics & Number $=6$ \\
\hline Intraoperative tumor location & 3 \\
Intrathymic & 2 \\
Aorto-pulmonary window & 1 \\
SVC-left brachiocephalic vein & \\
Postoperative biochemical level & 2.6 (range, $0.7-8.8)$ \\
PTH (pg/ml) & 2.2 (range, $2.0-2.4)$ \\
Calcium (mmol/ml) & 6 \\
Histology & 0 \\
Adenoma & 6 \\
Hyperplasia & 0 \\
\hline
\end{tabular}

$\mathrm{PTH}=$ parathyroid hormone; SVC = superior vena cava.

adenomas were intra-thymic in three patients, an aorto-pulmonary window in two patients, and one ectopic gland found at the junction of the left brachiocephalic vein and superior vena cava. All resected glands were confirmed to be parathyroid adenomas with a mean weight of 0.66 grams. Postoperative parathyroid hormones returned to normal and remained normal during the follow-up period (Table 2).

\section{Discussion}

The first successfully resected hyper-secretory mediastinal parathyroid adenoma was performed through a sternal-split in 1932 [1]. The majority of cases of primary hyperparathyroidism are caused by a single parathyroid adenoma. Most of the ectopic parathyroid adenomas are located in close proximity to the thymus gland. The inferior parathyroid glands originated from the third bronchial pouch within the thymus. Wang et al. reported a large series of 30 mediastinal parathyroid glands, 24 being intrathymic [2]. In our series, three patients' parathyroid adenomas were intrathymic. The incidence of mediastinal parathyroid adenoma is approximately $2 \%$ [3] [4]. In addition, mediastinal parathyroid adenomas are frequently small; therefore, when the adenoma is located deeper in the chest, preoperative localization studies are recommended. The decision regards which part of the chest to approach depends on preoperative localization imaging studies of the ectopic parathyroid adenoma. Several reports have noted that without preoperative assessment, the complication rate can be as high as $21 \%$ [5] [6] [7]. In our series, all patients with primary hyperparathyroidism had cervical ultrasound, dual phase Tc-99m sestamibi scintigraphy and enhanced CT scan of the neck and chest. Parathyroid adenomas are usually removed surgically through a cervical incision, but those located in the mediastinum are conventionally resected by median sternotomy or lateral thoracotomy [2] [8] [9]. These conventional procedures carry significant risk of injury to the phrenic nerve, laceration of the brachiocephalic vein, wound infection, and mediastinitis [10]. 
Conn et al. reported a complication rate of $19 \%$ after sternotomy for mediastinal parathyroidectomy [11]. Minimally invasive approaches to the mediastinum via video-assisted thoracic surgery and the robotic-surgical technique offer a significant decrease in the rate of complications compared to open sternotomy and thoracotomy. Video-assisted mediastinal parathyroidectomy was first reported by Prinz et al. in 1994. The rarities of deep mediastinal parathyroid adenoma explain the few reported cases treated with thoracoscopic and robotic-assisted techniques. Amer et al. reported seven cases of video-assisted parathyroid adenoma resection. The median hospital stay was two days and there was no operative mortality [12]. Tcherveniakov (2007) reported successful removal of mediastinal parathyroid adenoma using a video-assisted thoracic surgery technique without complications [13]. Robotic mediastinal parathyroidectomy constitutes the most recent addition in minimally invasive techniques. It is a logical evolution from conventional endoscopic surgery. Robotic systems enable the surgeon to work in a comfortable and ergonomic position: the eye and hand are in line with a 3-dimensional field of vision for depth of perception; instruments can be calibrated to improve dexterity. Published data on robotic-assisted mediastinal parathyroidectomy are limited to case reports in a small case series. Karagkounis et al. reported the experience with six patients who underwent robotic-assisted mediastinal parathyroidectomy. Median operative time was 168 minutes; mean pain score was 7.7 out of 10 on postoperative day 1 , and 1.5 out of 10 on postoperative day 10: the average hospital stay was 2.2 days [14]. One study series of five patients was reported by Ismail et al., with the mean operative time of 58 minutes, and the average hospital stay of 3 days, without any postoperative complications and no conversion to an open procedure, as all patients were free of symptoms during the 48 months of follow-up [15]. Our results revealed that all minimally invasive mediastinal parathyroidectomy patients were cured of their primary hyperparathyroidism, and the mean hospital stay was 3.8 days, but the operative time for robotic-assisted parathyroidectomy was found to be longer than video-assisted thoracoscopic parathyroidectomy, with the mean being 182 minutes versus 149 minutes. Other minimal access approaches included video-assisted mediastinoscopy more suitable for adenomas located in the anterior-superior mediastinum. Tcherveniakov et al. reported successful resection of ectopic mediastinal parathyroid adenoma in four patients using video-assisted mediastinoscopy [16]. We recommend the minimally invasive approach as a first line of treatment, particularly when the parathyroid adenoma is clearly located deeper than the brachiocephalic vein. Despite the positive results previously published in the literature, our opinion is that minimally invasive mediastinal parathyroidectomy is effective and safe, with low morbidity, when surgical expertise is available.

\section{References}

[1] Bauer, W. and Federman, D.D. (1962) Hyperparathyroidism Epitomized: The Case of Captain Charles E. Martell. Metabolism, 11, 21-29. 
[2] Wang, C., Gaz, R.D. and Moncure, A.C. (1986) Mediastinal Parathyroid Exploration: A Clinical and Pathologic Study of 47 Cases. World Journal of Surgery, 10, 687-695. https://doi.org/10.1007/BF01655558

[3] Ipponsugi, S., Takamori, S., Suga, K., et al. (1997) Mediastinal Parathyroid Adenoma Detected by $99 \mathrm{mTc}$-Methoxyisobutylisonitrile: Report of a Case. Surgery Today, 27, 80-83. https://doi.org/10.1007/BF01366946

[4] Prinz, R.A., Lonchyna, V., Carnaille, B., Wurtz, A. and Proye, C. (1994) Thoracoscopic Excision of Enlarged Mediastinal Parathyroid Glands. Surgery, 116, 999-1005.

[5] Wang, C., Gaz, R.D. and Moncure, A.C. (1986) Mediastinal Parathyroid Exploration: A Clinical and Pathologic Study of 47 Cases. World Journal of Surgery, 10, 68795. https://doi.org/10.1007/BF01655558

[6] Edis, A.J., Sheedy, P.F., Beahrs, O.H. and Van Heerdan, J.A. (1978) Results of Reoperation for Hyperparathyroidism with Evaluation of Preoperative Localization Studies. Surgery, 84, 384-393.

[7] Clark, O. (1988) Mediastinal Parathyroid Tumors. Archives of Surgery, 123, 1096-1100. https://doi.org/10.1001/archsurg.1988.01400330072011

[8] Russell, C.F., Edis, A.J., Scholz, D.A., Sheedy, P.F. and van Heerden, J.A. (1981) Mediastinal Parathyroid Tumors: Experience with 38 Tumors Requiring Mediastinostomy for Removal. Annals of Surgery, 193, 805-809.

https://doi.org/10.1097/00000658-198106000-00016

[9] Norton, J.A., Schneider, P.D. and Brennan, M.F. (1985) Median Sternotomy in Reoperations for Primary Hyperparathyroidism. World Journal of Surgery, 9, 807-813. https://doi.org/10.1007/BF01655200

[10] Hu, J., Ngiam, K.Y. and Parameswaran, R. (2015) Mediastinal Parathyroid Adenomas and Their Surgical Implications. Annals of the Royal College of Surgeons of England, 97, 259-261. https://doi.org/10.1308/003588415X14181254789088

[11] Conn, J.M., Goncalves, M.A., Mansour, K.A. and McGarity, W.C. (1991) The Mediastinal Parathyroid. American Surgeon, 57, 62-66.

[12] Amer, K., Khan, A.Z., Rew, D., Lagattolla, N. and Singh, N. (2015) Video Assisted Thoracoscopic Excision of Mediastinal Ectopic Parathyroid Adenomas: A UK Regional Experience. Annals of Cardiothoracic Surgery, 4, 527-334.

[13] Tcherveniakov, P., Menon, A., Milton, R., Papagiannopoulos, K., Lansdown, M. and Thorpe, J.A. (2007) Video-Assisted Mediastinoscopy (VAM) for Surgical Resection of Ectopic Parathyroid Adenoma. Journal of Cardiothoracic Surgery, 2, 41. https://doi.org/10.1186/1749-8090-2-41

[14] Karagkounis, G., Uzun, D.D., Mason, D.P., et al. (2014) Robotic Surgery for Primary Hyperparathyroidism. Surgical Endoscopy, 28, 2702-2707. https://doi.org/10.1007/s00464-014-3531-9

[15] Ismail, M., Maza, S., Swierzy, M., et al. (2010) Resection of Ectopic Mediastinal Parathyroid Glands with the da Vinci Robotic System. British Journal of Surgery, 97, 337-343. https://doi.org/10.1002/bjs.6905

[16] Tcherveniakov, P., Ashvini, M., Milton, R., Papagiannopoulos, K., Lansdown, M. and Thorpe, J. (2007) Video-Assisted Mediastinoscopy (VAM) for Surgical Resection of Ectopic Parathyroid Adenoma. Journal of Cardiothoracic Surgery, 2, 41. https://doi.org/10.1186/1749-8090-2-41 